\title{
Protein-Synthetic Machinery Beneath Postsynaptic Sites on CNS Neurons: Association Between Polyribosomes and Other Organelles at the Synaptic Site
}

\author{
Oswald Steward and Thomas M. Reeves \\ Departments of Neuroscience and Neurosurgery, University of Virginia School of Medicine, Charlottesville, Virginia 22908
}

Previous studies have demonstrated that polyribosomes are selectively positioned beneath postsynaptic sites on CNS neurons. In spine-bearing neurons, these polyribosomes are selectively localized at the base of the spines, and occasionally within spine heads. The present study evaluates whether there are relationships between the polyribosomes and other organelles of the postsynaptic cytoplasm, including membranous cisterns and spine apparatuses. Dendritic spines from the dentate gyrus and hippocampus of the rat were analyzed at the electron-microscopic level in 2 ways. First, relatively thick sections were prepared for electron microscopy, and spines were photographed in stereo using a goniometer stage. Second, conventional serial thin sections were taken, and spines were reconstructed. From the stereo photographs and serial reconstructions, we determined the proportion of polyribosomes that was associated with membranous cisterns. We also counted the number of ribosomes per cluster to determine whether there were differences between polyribosomes in different intradendritic locations, or between free polyribosomes and polyribosomes on cisternal membranes. From the serially reconstructed spines we determined the incidence of polyribosomes, membranous cisterns, and spine apparatuses, and evaluated the relationships between these organelles. We found that in both the dentate gyrus and hippocampus, about $50 \%$ of the polyribosomes that were present beneath the base of spines were associated with membranous cisterns. Polyribosomes that were present in the head of the spine were rarely associated with a cistern, however. The overall incidence of polyribosomes was similar in spines with spine apparatuses and spines without a spine apparatus. However, the polyribosomes that were present at the base of spines with a spine apparatus were much more likely to be on cisterns than were polyribosomes at the base of spines without an apparatus. These data suggest an interrelationship between the polyribosomes, membranous cisterns at the base of the spine, and spine apparatuses. We propose

\footnotetext{
Received Mar. 9, 1987; revised June 22, 1987; accepted June 25, 1987.

Thanks to P. M. Falk and S. L. Vinsant for their excellent technical help with the electron microscopy; to $\mathrm{P}$. Palmer for helping to develop the procedures using tannic acid; and to J. Gillenwater for help with the photography. Thanks also to William B. Levy for providing the collection of serial sections for analysis. Preparation of the serial sections was supported by NIH Grant NS 15488 to Dr. Levy. We also thank $R$. Lasek for suggesting the approach of counting the number of ribosomes per polyribosome. This work was supported by NIH Grant NS 12333 to O.S.

Correspondence should be addressed to Oswald Steward at the above address. Copyright (C) 1988 Society for Neuroscience $0270-6474 / 88 / 010176-09 \$ 02.00 / 0$
}

that these organelles may represent different components of a system for the synthesis and processing of proteins related to the synaptic site.

Recent studies have revealed that protein-synthetic machinery comprised of polyribosomes and membranous cisterns is selectively positioned beneath postsynaptic sites on CNS neurons. In spine-bearing neurons, most of the polyribosomes that are present in dendrites are positioned at the base of dendritic spines (Steward and Levy, 1982). Polyribosomes are also localized beneath non-spine synapses, such as the ones on axon initial segments (Steward and Ribak, 1986). The polyribosomes are particularly prominent during times of synapse growth (Steward, 1983a, b; Steward and Falk, 1985, 1986; and see Palacios-Pru et al., 1981). The positioning of polyribosomes at the synaptic site provides a mechanism whereby some of the key molecules of the synaptic junction can be synthesized locally.

While polyribosomes seem to be a ubiquitous component of many types of CNS synapses, questions remain about the nature of the synthetic apparatus and its association with other organelles at the synaptic site. First, qualitative observations reveal that the polyribosomes are sometimes very near membranous cisterns. This is of interest, since polyribosomes that are associated with membranes usually synthesize a different class of proteins than do free polyribosomes; membrane proteins and proteins for release from the cell are usually synthesized on membrane-bound ribosomes (rough endoplasmic reticulum), whereas proteins for intracellular use (cytoplasmic and cytoskeletal proteins, soluble cnzymcs, ctc.) arc usually synthesized on free polyribosomes (Palade, 1965). While there are exceptions to the generalizations regarding the types of proteins produced by membrane-bound polyribosomes (Hall et al., 1982; Fujiki et al., 1984), information about the association between ribosomes and cisterns at least provides clues about the types of proteins being synthesized.

Second, while most of the polyribosomes that are present in spine-bearing dendrites are positioned under the spine base, there are some polyribosomes in other locations, including the shaft of the dendrite, and the spine neck or head. Thus, we were interested in determining whether there were any differences between polyribosomes in the different intradendritic locations that might indicate differences in the types of proteins being produced.

Third, qualitative observations suggest that the polyribosomes and associated cisterns sometimes are contiguous with a spine apparatus. It has been suggested that the spine apparatus plays some role in synapse remodeling (Tarrant and Routtenberg, 1979; Dyson and Jones, 1984); in this regard, an interesting 
possibility is that it might be involved in posttranslational processing or the transport of proteins to the synaptic site (Tarrant and Routtenberg, 1977, 1979; Manina, 1979; Westrum et al., 1980; Spacek, 1985a). Thus, we were interested in determining whether polyribosomes were colocalized with spine apparatuses, and, if so, what the relationship between these organelles might be.

Finally, qualitative observations have suggested that there might be a fairly uniform number of singlet ribosomes in the polyribosomes. The number of ribosomes in a polyribosome is often related to the length of the messenger RNA (Warner et al., 1962). Thus, the number of ribosomes could provide a clue about the size of the proteins being synthesized. For this reason, we wished to determine whether there were differences in the number of ribosomes per polyribosome in different intradendritic locations.

To address these questions, the present study quantitatively analyzes dendritic polyribosomes and their associated organelles at the electron-microscopic level by stereoviewing of relatively thick sections and serial reconstruction of conventional thin sections. Our results revealed that (1) about half of the polyribosomes beneath spines were associated with membranous cisterns. The few polyribosomes that were found in spine heads were almost always free, however; (2) spines with spine apparatuses were much more likely to have polyribosomes on cisterns than were spines without an apparatus; (3) there were some differences in the average number of ribosomes per cluster in different intradendritic locations. Some of these data have been summarized in reviews (Steward, 1983a, b; Steward et al., in press).

\section{Materials and Methods}

Experimental animals were adult male Sprague-Dawley rats, ranging in weight from 200 to $300 \mathrm{gm}$. Most of the observations were carried out in the dentate gyrus, because there is already a substantial data base on polyribosomes beneath synaptic sites on dentate granule cells (Steward and Levy, 1982). Limited observations were also carried out in the CA1 region of the hippocampus proper for comparison. We used 2 approaches to obtain 3-dimensional information about the polyribosomes beneath synapses and their association with other organelles of the subsynaptic cytoplasm. First, we used stereoviewing techniques to evaluate relatively thick sections. This approach offers the advantage that a reasonable number of dendritic polyribosomes can be evaluated fairly easily, but has the disadvantage that adjacent structures are lost as a result of the section preparation. For this reason, the thick sections could not be used to evaluate whether polyribosomes and spine apparatuses were codistributed. Thus, as a second approach, a limited number of synapses were completely reconstructed from serial sections.

Preparation of material for stereoviewing. For studies of the association of polyribosomes with cisterns, we found it useful to perfuse animals with fixatives containing tannic acid. Tannic acid is thought to stabilize elements of the cytoskeleton, and adds considerably to membrane contrast. A number of different fixation protocols were tried, and best results were obtained when the perfusion was initiated with $4 \%$ glutaraldehyde in either phosphate or cacodylate buffer, followed by glutaraldehyde/tannic acid solutions in which tannic acid concentration ranged between 0.1 and $0.2 \%$. The material that was analyzed in detail in the present study was obtained from an animal perfused with 100

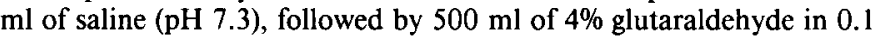
M phosphate buffer (pH 7.0), and then $1000 \mathrm{ml}$ of $2 \%$ glutaraldehyde/ $0.1 \%$ tannic acid (Mallinkrodt) in 0.1 , $\mathrm{M}$ phosphate buffer ( $\mathrm{pH} 7.0$ ).

The brains were allowed to postfix in situ for $4 \mathrm{hr}$, and were then sectioned on a Vibratome at $200 \mu \mathrm{m}$. The hippocampal formation was dissected free from sections taken at about $1.5 \mathrm{~mm}$ posterior to the anterior boundary of the hippocampus (i.e., the fifth or sixth Vibratome section posterior to the first section that contained gray matter of the hippocampus). The sections were then osmicated using routine procedures, and embedded in Maraglas. Sections with purple interference colors (estimated thickness, $150-200 \mathrm{~nm}$ ) were cut roughly parallel with the long axis of the dendrites of the cells being evaluated.

The sections were examined using a Jeol 100CX microscope equipped with a goniometer stage. The middle molecular layer of the dentate gyrus and middle stratum radiatum of the CAl region of the hippocampus were scanned for clusters of ribosomes in dendrites (either under spines or within the dendrite proper). When a cluster was encountered, stereo photographs were taken at a $10^{\circ}$ tilt angle (magnification, 20,000 $\times$ ). A cluster of ribosomes was considered a polyribosome when 3 or more ribosomes were present and when the spacing between ribosomes was comparable to that of polyribosomes in the cell body. One hundred twenty-two pairs of photographs were taken from the dentate gyrus, yielding a total of 289 polyribosomes (most photographs had more than one polyribosome); 74 pairs of photographs were taken in the CAl region of the hippocampus proper, yielding 129 polyribosomes.

From the stereo photographs, we first defined the location of all polyribosomes (whether they were beneath identified or presumed spine bases, within the spine neck or head, or within the dendrite proper Identified spine bases are those where the spine and its attachment to the parent dendrite are visible in their entirety. Presumed spine bases are "mounds" that resemble the base of spines that extend out of the plane of the section. Serial reconstructions have previously revealed that most such "mounds" are in fact the base of spines (Steward and Levy, 1982). Second, we determined whether the polyribosomes were associated with a cisternal membrane. Polyribosomes were considered to be associated with a cistern if they were situated within 1 microtubule diameter (about $20 \mathrm{~nm}$ ) of a cistern. Finally, we counted the individual ribosomes in the cluster.

Preparation of material for serial reconstruction. The tissue that was used for the serial section analysis has been described previously (Steward and Levy, 1982). Originally, this tissue was prepared as the control material for an analysis of changes in spine shape following the induction of long-term potentiation (I.TP). An animal was prepared for acute neurophysiological recording, LTP was induced unilaterally in the temporodentate system, and the animal was then perfused with $2 \%$ paraformaldehyde $/ 2 \%$ glutaraldehyde in $0.13 \mathrm{~m}$ sodium cacodylate buffer (pH 7.2). Twenty serial sections were obtained from the middle molecular layer of the dentate gyrus contralateral to the stimulation. From this series, an area of approximately $210 \mu \mathrm{m}^{2}$ was reconstructed as a montage of 4 photographs taken at an initial magnification of $10,000 \times$, covering an area of about $7 \times 30 \mu \mathrm{m}$ (the size varied slightly from montage to montage owing to differences in the overlap of the composite photographs). In our initial analysis (Steward and Levy, 1982), 34 through-sectioned spines were identified and reconstructed. The series was reanalyzed for the present study, and an additional 84 throughsectioned spines were identified and reconstructed for a total sample of 118 spines. Of these, 93 were reconstructed in their entirety, and 25 were reconstructed sufficiently to permit an analysis of any polyribosomes that were present.

In the serial sections, we focused exclusively on polyribosomes that were at the base of spines or within the spine itself. Two or more ribosomes with a spacing consistent with polyribosomes were considered a polyribosome; 45 such polyribosomes were identified. As for the stereo photographs, we determined whether the polyribosomes were associated with a cisternal membrane, and we counted the number of ribosomes in the cluster, summing across sections. No correction factor was applied for double-counting. This should not introduce a major error, since ribosomes are small (about $15 \mathrm{~nm}$ ) relative to section thickness $(60-70 \mathrm{~nm})$. In addition, we evaluated all spines for spine apparatuses to determine whether polyribosomes and spine apparatuses were associated.

\section{Results}

As noted in previous studies, it is apparent in fortuitous thin sections that some of the polyribosomes that are present beneath dendritic spines are associated with membranous cisterns. The precise nature of the association is difficult to evaluate in conventional thin sections, however, since it is not possible to define 3-dimensional relationships. In stereo photographs of 150-200nm-thick sections (Fig. 1), it becomes evident that many of the polyribosomes cluster about tubular sacules of endoplasmic reticulum; often, the polyribosomes appear to form a cap around 
Figure 1. Examples of dendritic spines with underlying polyribosomes on membranous cisterns. This stereo pair shows spines from the molecular layer of the dentate gyrus. Two clusters of ribosomes are evident (arrows); both appear to cap the terminus of a membranous cistern. $s$, Spine head; den, dendrite. Magnification, $\times 30,000$.
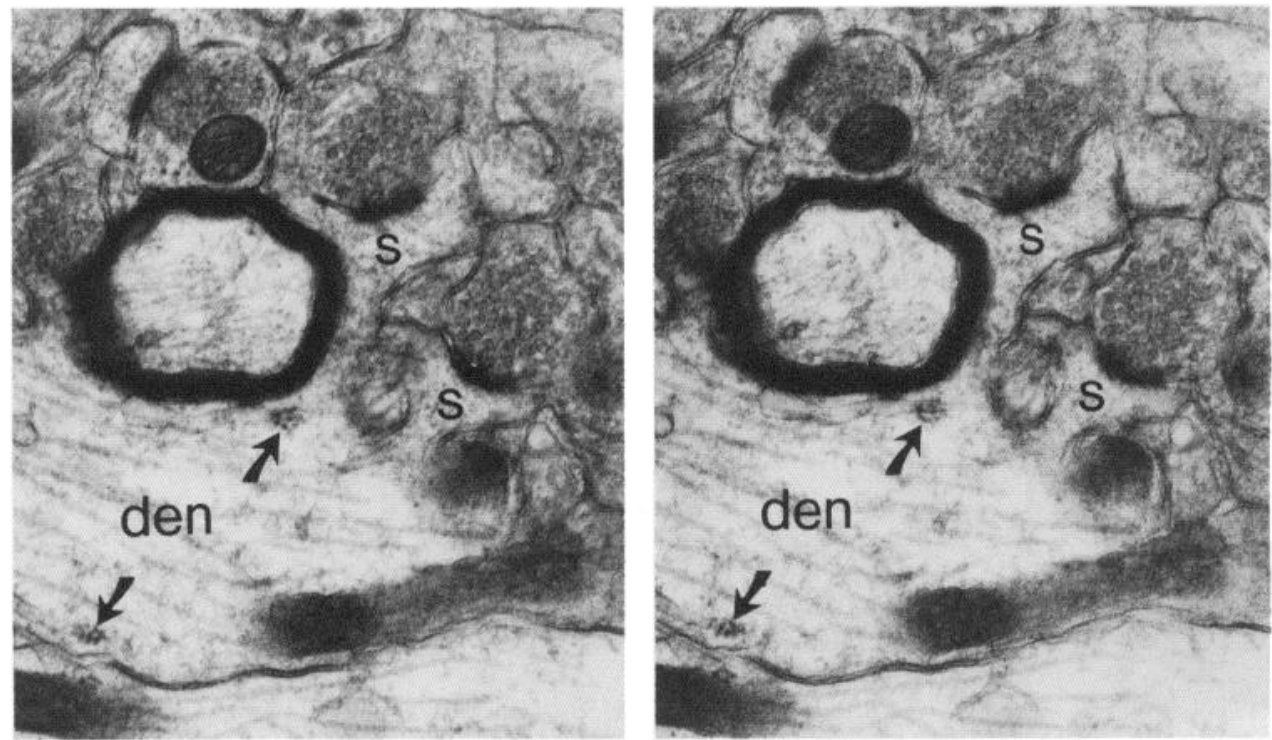

the terminus of a cistern. Sometimes, polyribosomes can be found on cisterns that appear to be connected to a spine apparatus (Fig. 2).

Quantitative analysis of the association between polyribosomes and other organelles in the subsynaptic cytoplasm

Table 1 summarizes the data from the analyses of the stereo photographs. Of the 289 polyribosomes that were identified in the middle molecular layer of the dentate gyrus, $179(62 \%)$ were associated with spines; 44 of these were in the base of identified spines, 129 were beneath mounds (presumed spine bases), and 6 were in the spine head. Of the total, about half $(47 \%)$ lay within 1 microtubule diameter (about $20 \mathrm{~m}$ ) of a cistern. The values were similar $(52 \%)$ when combining the categories of identified and presumed spines. When considering only the polyribosomes at the base of identified spines, $64 \%$ were associated with a cistern. A test of differences between proportions (Bruning and Kintz, 1977) revealed that none were significant ( $z$ ranged from 0.24 to 1.83 ; significance at the 0.05 level requires a $z$ of
1.96). Interestingly, only 1 of the $6(17 \%)$ polyribosomes that were present in the spine head was associated with a cistern. This proportion was significantly different from the value of $64 \%$ from the "identified spine" category $(z=2.19 ; p<0.05)$, but not from the value of $48 \%$ from the category of presumed spines $(z=1.49)$ or from the value of $52 \%$ from the combined category of identified and presumed spines $(z=1.69)$. The reliability of these comparisons is questionable, however, because of the small number of polyribosomes in spine heads. As illustrated in Table 1B, the data for the CA1 region of the hippocampus were similar. Again, none of the differences between proportions were significant ( $z$ ranged from 0.17 to 0.70 ).

While stereoviewing of single thick sections provides an indication of whether polyribosmes are apposed to cisternal membranes, this method is not adequate to evaluate whether polyribosomes are codistributed with spine apparatuses. The problem is that spines may contain polyribosomes and a spine apparatus without both organelles being visible in any given section. In addition, because the cisternae with which the polyribosomes
Figure 2. Dendritic spine with a spine apparatus and underlying polyribosomes on a membranous cistern. Note that the polyribosomes (arrow) appear to be closely associated with the cisternal membrane at this point. The tubular cistern can be seen to curve into the depth of the section and intersect the surface of the section at its upper limit. A spine apparatus can be seen to the left of the arrow. Abbreviations as in Figure 1.
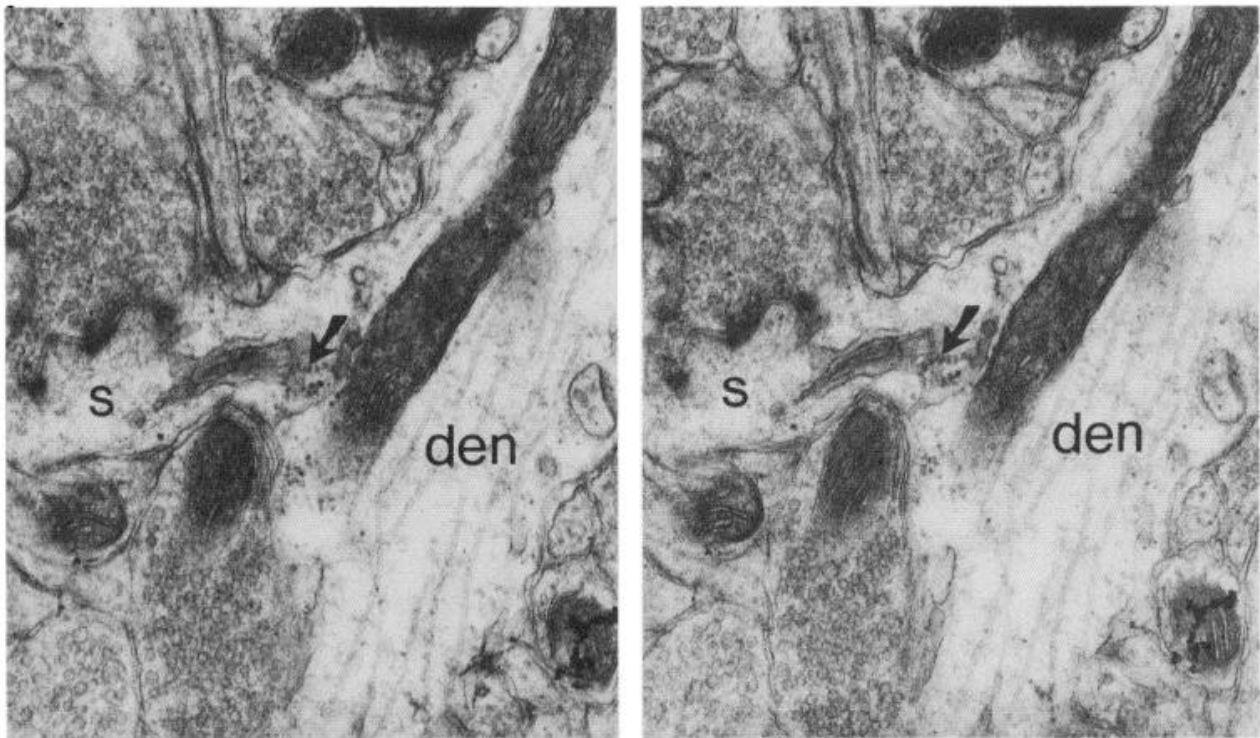

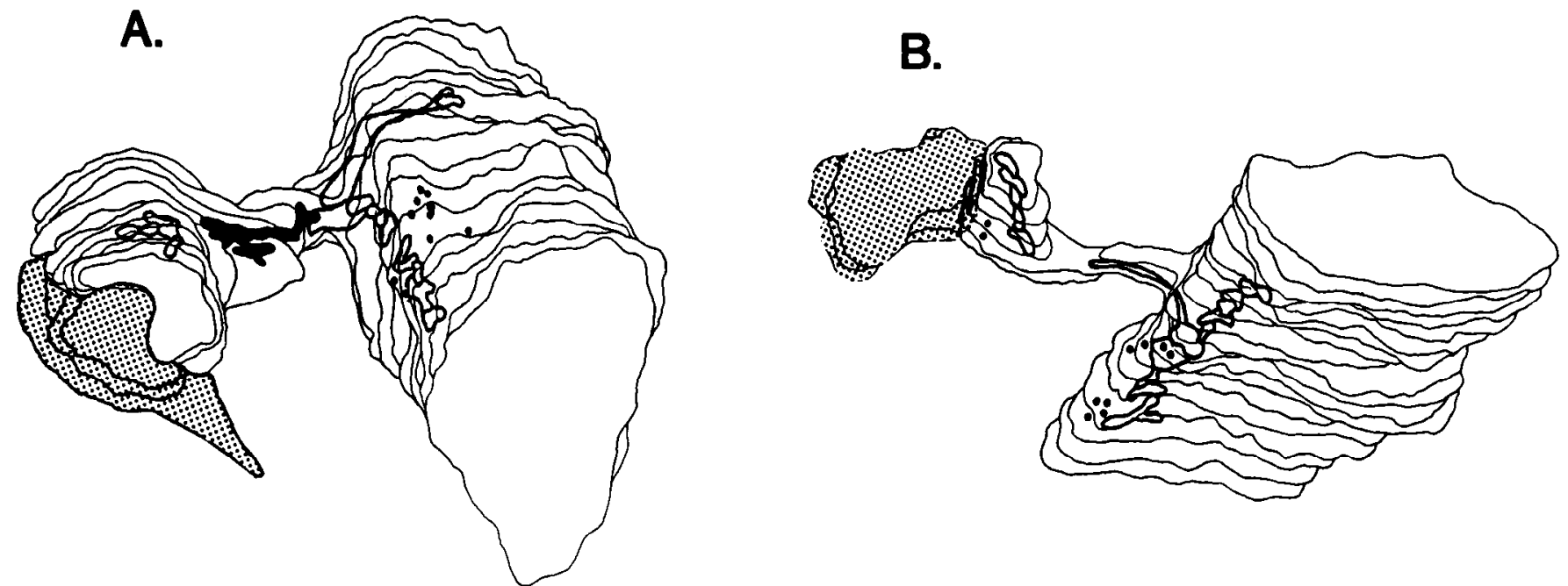

Figure 3. Serial reconstruction of dendritic spines. Two spines are reconstructed. The sections were offset slightly during alignment so as to convey an impression of 3-dimensionality. The actual section alignment may be different than shown. $A$, The spine has a spine apparatus (heaviest line) and cisterns beneath the spine base and in the spine head (medium lines). $B$. The spine has a cistern in the spine head and at the spine base, but no spine apparatus. Dots indicate ribosomes.

were associated were often tubular, we were concerned that some of the cisterns associated with particular clusters could lie out of the plane of the section containing the polyribosomes. If this were true, our analysis of thick sections might underestimate the actual association between polyribosomes and cisterns. For these reasons, the association between polyribosomes and other organelles was evaluated by reconstructing spines from serial sections. Because there was no obvious difference between dentate granule cells and hippocampal pyramidal cells, we carried out the serial section reconstructions in the dentate gyrus only.

\section{Table 1. Analysis of stereo photographs of thick sections}

\begin{tabular}{llll}
$\begin{array}{l}\text { Location of } \\
\text { polyribosomes }\end{array}$ & $\begin{array}{l}\text { No. of } \\
\text { polyribo- } \\
\text { somes }\end{array}$ & $\begin{array}{l}\text { No. on } \\
(\%)\end{array}$ & $\begin{array}{l}\text { cisterns } \\
\text { ribosomes no. of singlet }\end{array}$ \\
\hline
\end{tabular}

A. Polyribosomes in dendrites of dentate granule cells

$\begin{array}{lccl}\text { Identified } & 44 & 28 & 7.4 \pm 3.2 \text { on cisterns } \\ \quad \text { spine base } & & (64) & 7.2 \pm 2.9 \text { free } \\ \text { Presumed } & 129 & 62 & 8.5 \pm 4.0 \text { on cisterns } \\ \quad \text { spine base } & & (48) & 8.2 \pm 4.4 \text { free } \\ \text { Spine head } & 6 & 1 & 4 \text { on cistern } \\ & & (17) & 5.8 \pm 1.8 \text { free } \\ \text { Other } & 110 & 46 & 9.8 \pm 5.9 \text { on cisterns } \\ & & (42) & 8.8 \pm 5.0 \text { frec } \\ \text { Totals } & 289 & 137 & \\ & & (47)\end{array}$

B. Polyribosomes in dendrities of hippocampal pyramidal cells

$\begin{array}{lccl}\text { Identified } & 24 & 10 & 7.5 \pm 3.8 \text { on cisterns } \\ \quad \text { spine base } & & (42) & 8.5 \pm 3.1 \text { free } \\ \text { Presumed } & 55 & 22 & 8.0 \pm 4.1 \text { on cisterns } \\ \quad \text { spine base } & & (40) & 7.7 \pm 3.1 \text { free } \\ \text { Spine head } & 1 & 0 & 6 \text { free } \\ & & (0) & \\ \text { Other } & 59 & 28 & 6.8 \pm 3.6 \text { on cisterns } \\ & & (47) & 7.0 \pm 3.9 \text { free } \\ \text { Totals } & 139 & 60 & \\ & & (43) & \end{array}$

Examples of serial reconstructions of spines and their organelles are illustrated in Figure 3.

Table 2 summarizes the data from the analysis of $93 \mathrm{com}$ pletely reconstructed spines, and from 25 spines that were reconstructed sufficiently for evaluating polyribosomes that were present. A total of 30 spines (25\%) had polyribosomes at the base; a few spines had more than one cluster, so that the total number of polyribosomes beneath spine bases was 36 . Nine spines $(7 \%)$ had polyribosomes in the head; $53 \%$ of the polyribosomes at the base of the spine were associated with cisterns; whereas none of the polyribosomes in spine heads were associated with a cistern. In part, these data may reflect the distribution of cisterns: while $83 \%$ of the total number of spines had cisterns, $47 \%$ of the spines with free polyribosomes at the base did not. In addition, cisterns were uncommon in spine heads. The distribution of cisterns cannot be the entire story, however, because $53 \%$ of the spines with free polyribosomes at the base also had a cistern.

\section{Evaluation of the relationship between polyribosomes and spine apparatuses}

The data analyzing the degree of association between polyribosomes and spine apparatuses are summarized in Table 3. Twenty-nine of the 118 spines had a spine apparatus $(25 \%)$, but only 10 of the $29(34 \%)$ had polyribosomes at the base. In comparison, 20 of the 89 spines without a spine apparatus had polyribosomes at the base (22\%). The differences between proportions were not significant $(z=1.30)$. Thus, 20 spines had

Table 2. Analysis of serial sections

\begin{tabular}{llcl}
$\begin{array}{l}\text { Location of } \\
\text { polyribosomes }\end{array}$ & $\begin{array}{l}\text { No. of } \\
\text { polyribo- } \\
\text { somes }\end{array}$ & $\begin{array}{l}\text { No. on } \\
\text { cisterns } \\
(\%)\end{array}$ & $\begin{array}{l}\text { Mean no. of singlet } \\
\text { ribosomes }\end{array}$ \\
\hline Spine base & 36 & 19 & $8.3 \pm 3.5$ on cistern \\
& & $(53)$ & $8.1 \pm 7.2$ free \\
Spine head & 9 & 0 & $4.2 \pm 1.5$ free \\
& & $(0)$ &
\end{tabular}


Table 3. Incidence of polyribosomes at spine bases with and without spine apparatuses ${ }^{a}$

\begin{tabular}{lccc} 
& $\begin{array}{l}\text { Total } \\
\text { spines } \\
(\%)\end{array}$ & $\begin{array}{l}\text { Spines } \\
\text { with S.A. } \\
(\%)\end{array}$ & $\begin{array}{l}\text { Spines } \\
\text { without } \\
\text { S.A. } \\
(\%)\end{array}$ \\
\hline Number & 118 & 29 & 89 \\
& & $(25)$ & $(75)$ \\
Number with polyribosomes & 30 & 10 & 20 \\
& $(25)$ & $(34)$ & $(22)$ \\
Spines with polyribosomes & 16 & 8 & 8 \\
$\quad$ on cisterns & $(14)$ & $(28)$ & $(9)$ \\
Spines with free & 15 & 2 & 13 \\
polyribosomes & $(13)$ & $(7)$ & $(15)$ \\
Spines with cisterns & 98 & 27 & 71 \\
& $(83)$ & $(93)$ & $(78)$ \\
\hline
\end{tabular}

a Polyribosomes with spine heads are not included in this analysis.

polyribosomes but no spine apparatus, 19 had a spine apparatus but no polyribosomes, and only 10 spines had both. There were also no special associations betwecn spinc apparatuses and polyribosomes in spine heads. Two out of the 29 spines with spine apparatuses and 7 out of 89 spines without apparatuses had polyribosomes in the head ( 7 and $8 \%$, respectively).

There was evidence of a special relationship between spine apparatuses and polyribosomes on cisterns. As illustrated in line 3 of Table 3,8 out of the total of 29 spines with a spine apparatus $(28 \%)$ had polyribosomes at the base that were on a cistern, whereas in the case of spines without spine apparatuses, only 8 out of $88(9 \%)$ had polyribosomes on cisterns. These differences were significant $(z=2.59 ; p<0.01)$. Table 4 illustrates the relationship between polyribosomes on cisterns and spine apparatuses from a different perspective. Of the polyribosomes that were present at the base of spines with a spine apparatus, 10 of $12(83 \%)$ were associated with a cistern. In contrast, only 9 out of $24(38 \%)$ of the polyribosomes at the base of spines without a spine apparatus were associated with a cistern. The difference between these proportions was significant $(z=2.55$; $p<0.025$ ), as was the difference in the incidence of free polyribosomes in spines with and without spine apparatuses (17 vs $62 \% ; z=2.55 ; p<0.025)$.

The greater frequency of polyribosomes on cisterns beneath spines with spine apparatuses could come about if spines with a spine apparatus were more likely to have underlying cisterns. The final line of Table 3 addresses this question. Twenty-seven out of $29(93 \%)$ of the spines with spine apparatuses had cisterns, whereas 71 out of $89(78 \%)$ of the spines without spine apparatuses had cisterns. These differences approached significance $(z=1.81)$. Nevertheless, they were not large enough to account for the 2-fold difference in the incidence of cistern-bound polyribosomes.

\section{Analysis of the number of ribosomes per polyribosome}

Tables 1 and 2 also summarize the counts of the number of ribosomes per polyribosome in the different intradendritic locations. In general, the number of ribosomes per polyribosome under identified and presumed spine bases and elsewhere in the dendrites was comparable. However, the data from both stereoviewing and serial reconstruction indicate that there were fewer ribosomes per cluster in the case of the polyribosomes in the spine head.
Table 4. Association between polyribosomes and cisterns at spine bases with and without spine apparatuses ${ }^{a}$

\begin{tabular}{lcc} 
& $\begin{array}{l}\text { Spines with } \\
\text { S.A. (\%) }\end{array}$ & $\begin{array}{l}\text { Spines } \\
\text { without } \\
\text { S.A. (\%) }\end{array}$ \\
\hline Total polyribosomes & 12 & 24 \\
Number of polyribosomes & 10 & 9 \\
$\quad$ on cisterns & $(83)$ & $(38)$ \\
Number of free & 2 & 15 \\
polyribosomes & $(17)$ & $(62)$ \\
\hline Polyribosomes within spine heads are not included in this analysis.
\end{tabular}

${ }^{2}$ Polyribosomes within spine heads are not included in this analysis.

As we were analyzing these data, it became clear that a simple calculation of the average number of ribosomes per cluster was not appropriate. In some cases, relatively large clusters of ribosomes were present, and at times it was not clear whether these represented one large cluster or 2 or more small clusters lying close together. One way to evaluate this situation is to make use of frequency histograms of the incidence of variously sized clusters. If there is, on the average, a consistent number of individual ribosomes in a polyribosome, and if 2 or more clusters are sometimes found together, then frequency histograms ought to reveal one peak that corresponds to one polyribosome, a smaller peak corresponding to 2 polyribosomes, and so on. Alternatively, if there is a population of large polyribosomes, then these could contain any number of ribosomes.

Frequency histograms of the number of ribosomes per cluster in the dentate gyrus are illustrated in Figures 4 and 5 . Figure 4 presents the frequency histograms from the analyses of the thick sections for polyribosomes beneath identified spines (Fig. 4A), beneath mounds (Fig. 4B), and in the dendrite proper (Fig. 4C). The mode of the distribution in all 3 locations was 5 , and there was no obvious difference between the frequency histograms from the various locations except that there appeared to be more large polyribosomes in the dendrite proper.

$\Lambda$ s we suggested above, most "mounds" are actually the base of spines, and thus the categories of identified (Fig. 4A) and presumed (Fig. $4 B$ ) spines can be combined (Fig. $4 C$ ). With the increased data base, the distribution becomes somewhat smoother, but its form is not altered substantially. Again, there were more large polyribosomes in the dendrite proper than in the combined category of identified and presumed spines. Indeed, only 4 out of the total of 172 clusters $(2 \%)$ in the combincd category had more than 18 ribosomes, whereas in the dendrite proper, 11 out of 110 clusters $(10 \%)$ had more than 18 ribosomes. This difference in proportion is significant $(z=2.97$; $p<0.005$ ). It is interesting that 2 of the large clusters in the dendrite proper were found at dendritic branch points. In all of the histograms, but particularly in the combined "identified and presumed spine" category (Fig. $4 D$ ), the peak in the frequency histogram is at $5-8$ ribosomes per cluster, but there is a small secondary peak at 14-16 ribosomes, as would be expected if some of the large clusters were actually 2 or more smaller clusters lying together.

The counts of ribosomes in single thick sections are potentially compromised because clusters are large with respect to section thickness, and thus some of the ribosomes of given clusters could lie out of the plane of a single section. A comparison of the mean number of ribosomes per cluster between thick sections (Table 1) and serial reconstructions (Table 2) suggests that this 
A.

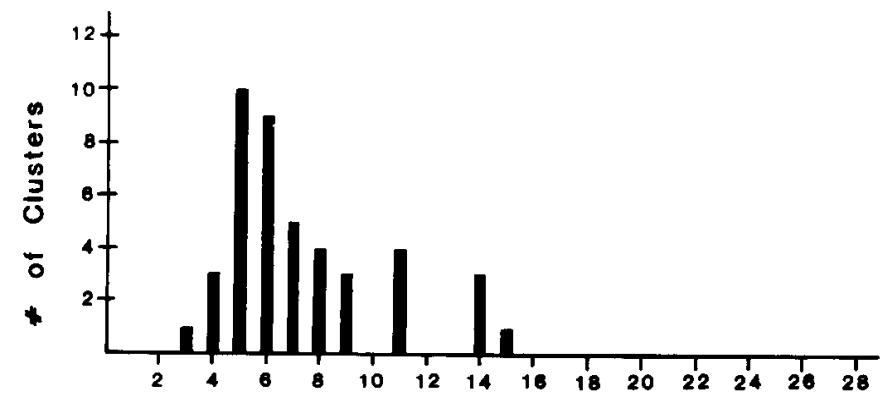

B.

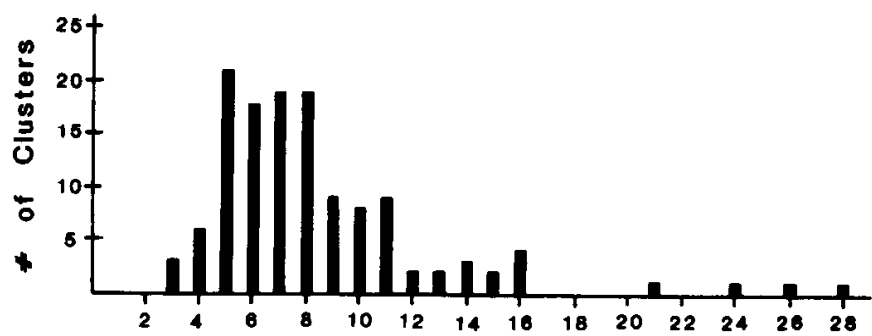

C.

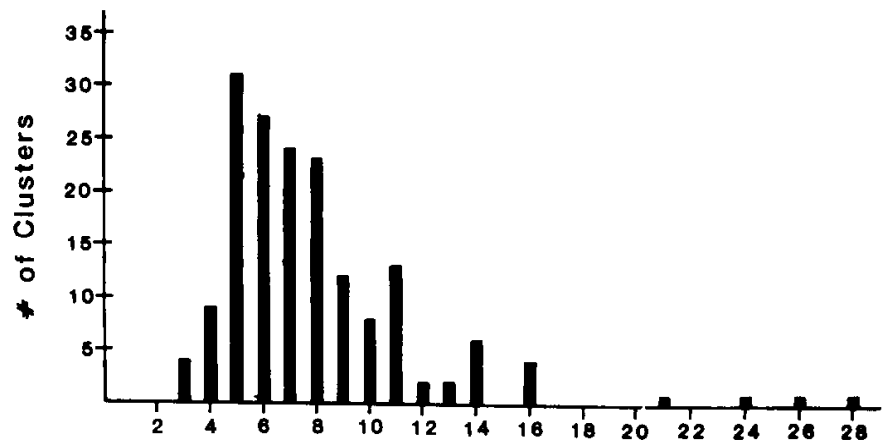

D.

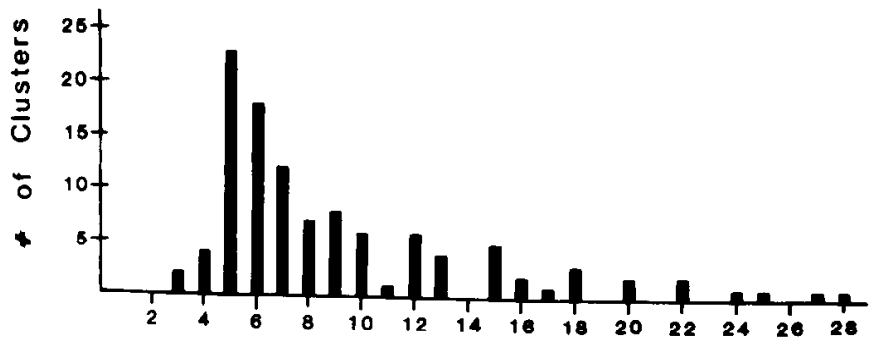

E.

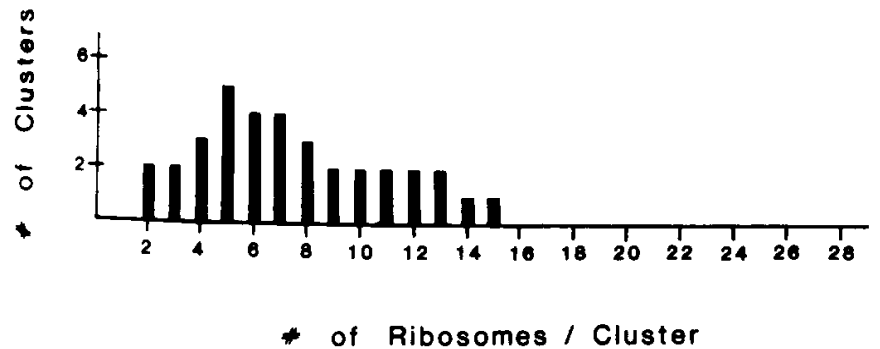

Figure 4. Frequency histograms of the number of ribosomes per cluster from the molecular layer of the dentate gyrus. $A$, Polyribosomes bencath identificd spines. $B$, Polyribosomes beneath presumed spines (mounds). $C$, Polyribosomes beneath either identified or presumed spines ( $A$ and $B$ combined). $D$, Polyribosomes within the dendrite proper that are not obviously associated with spines. $E$, Polyribosomes beneath spines in the serially reconstructed sample. ( $C$ and $D$ are redrawn from Steward, 1983b.) may not be a major problem, but it was still of interest to determine if the form of the frequency histogram would be different when considering counts that werc not subject to attenuation. Figure $4 E$ shows the frequency histogram of the counts of ribosomes beneath spines, as obtained from the serial reconstructions. The modal number of ribosomes per cluster was again 5 , and the principal peak in the frequency histogram was at 5-8 ribosomes. Of course, the total number of clusters was 
A.

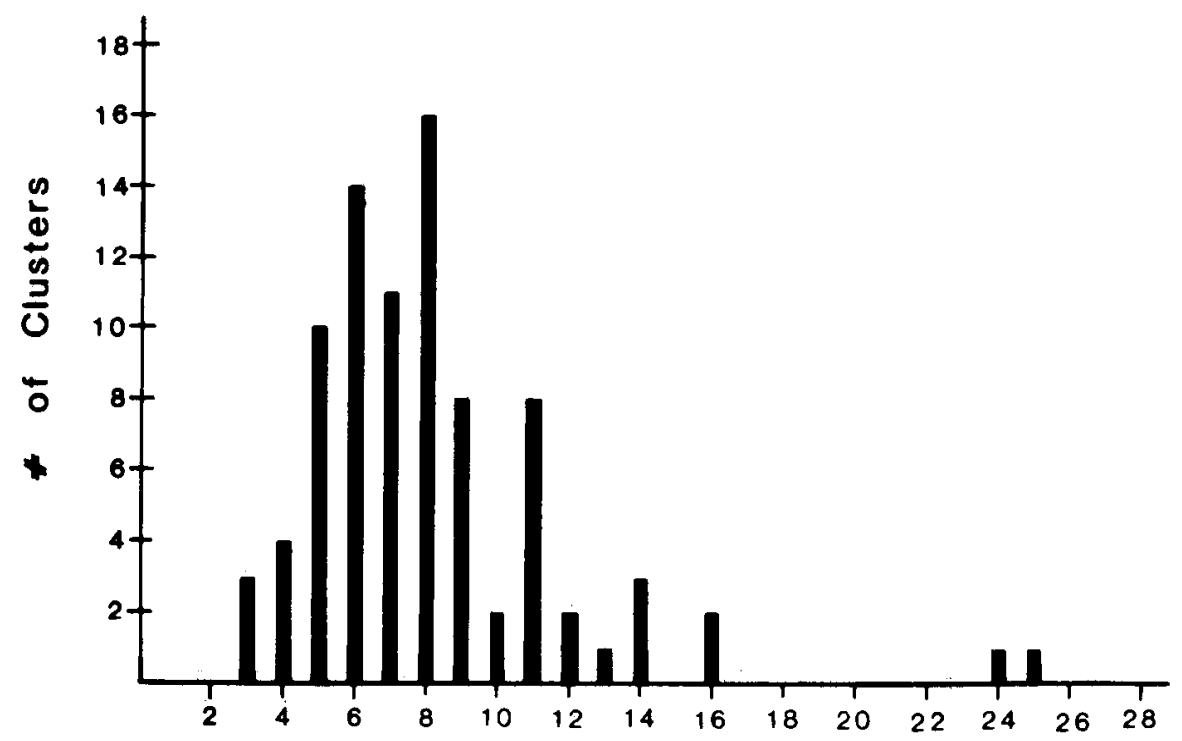

Figure 5. Frequency histograms of the number of ribosomes in cistern-associated and free polyribosomes (dentate gyrus). Only the polyribosomes beneath identified or presumed spines are evaluated in these graphs. $A$, Polyribosomes on cisterns. $B$, Free polyribosomes. (Redrawn from Steward, 1983b.)

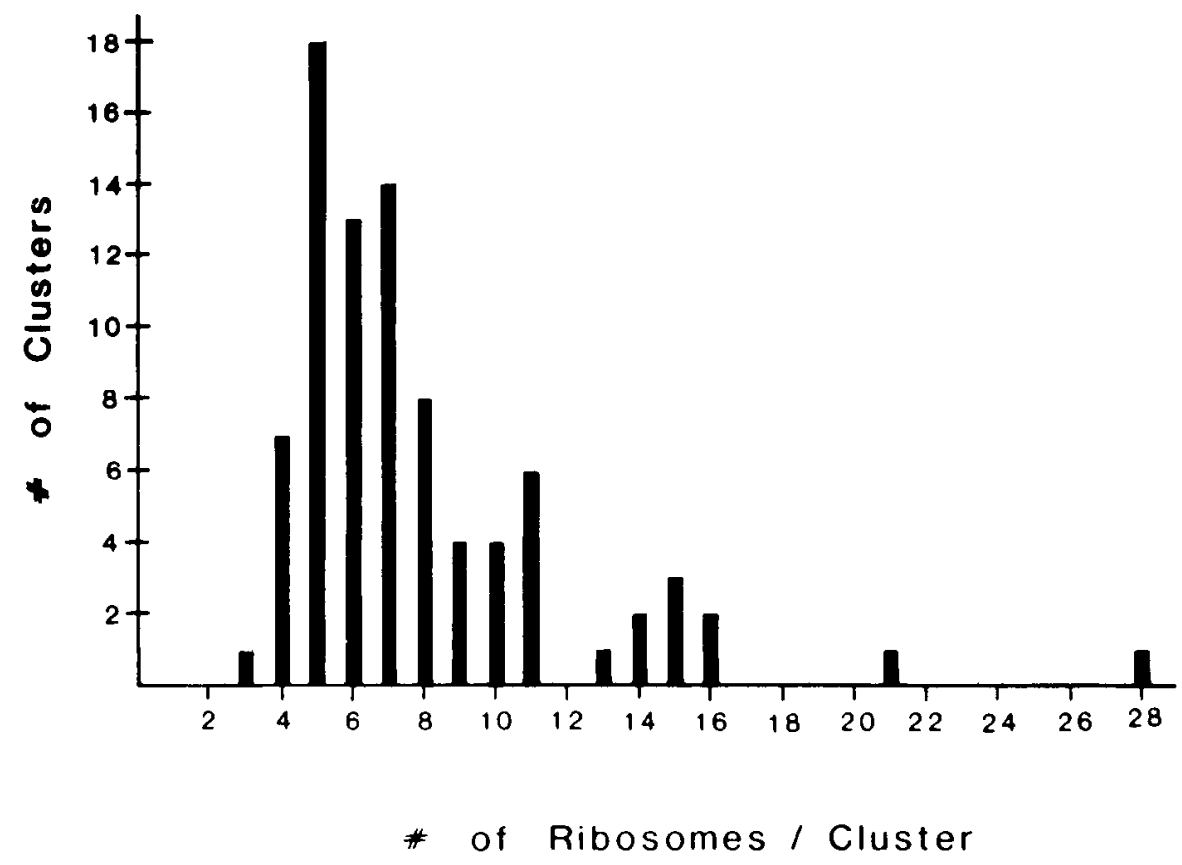

smaller, and thus the data are somewhat less reliable than for the counts from the thick sections. Nevertheless, it is clear that there were no major differences in the distributions when comparing data obtained from stereoviewing of thick sections (Fig. $4 D$ ) with the data from serial reconstructions (Fig. $4 E$ ).

In Figure 5, the polyribosomes beneath identified or presumed spines are further analyzed according to whether the ribosomes arc on a cistern or frec. It is interesting that for the population of polyribosomes on cisterns, the modal number of ribosomes per cluster was 8 (compare with other frequency histograms of Fig. 4). The form of the histogram was also less skewed than those of Figure 4. On the other hand, the histogram for the free polyribosomes was quite comparable to those of Figure 4; again, the modal number of ribosomes per cluster was 5 , with the peak of the distribution occurring between 5 and 8 ribosomes. A secondary peak in the frequency histogram was apparent between 13 and 16 ribosomes per cluster.

Figures 6 and 7 show frequency histograms for the number of ribosomes per polyribosome in the CA1 region of the hippocampus proper. The frequency histogram for the polyribosomes under identified and presumed spine bases (Fig. 6A), and the frequency histogram for the dendritic polyribosomes that are not apparently associated with spines (Fig. $6 B$ ) are comparable to the histograms from the dentate gyrus (see Fig. 4, $B$, $C)$. Analyzing the spine-associated polyribosomes according to whether the ribosomes are near a cistern or free, one finds that the histogram for free polyribosomes is virtually identical to that from the dentate gyrus (compare Fig. $7 A$ with Fig. $5 A$ ). However, in the histogram for the polyribosomes associated with cisterns, the modal number of ribosomes on cisterns is 5 , and this histogram is skewed like the histogram for free polyribosomes (Fig. $7 B$ ). This is somewhat different than the comparable histogram for dentate granule cells (compare Fig. $7 B$ with Fig. 5B). 
A.

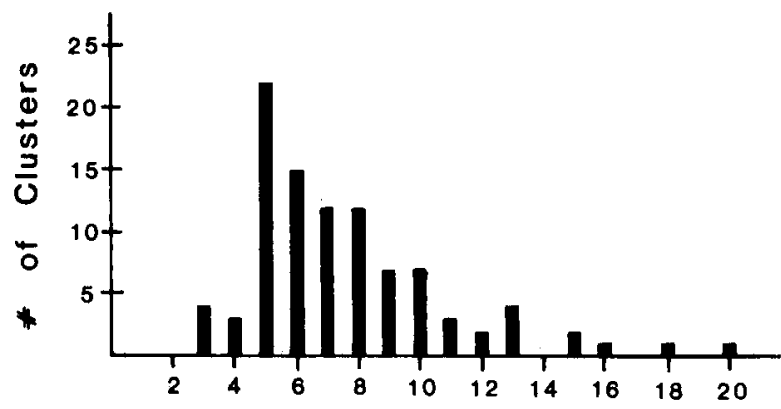

B.

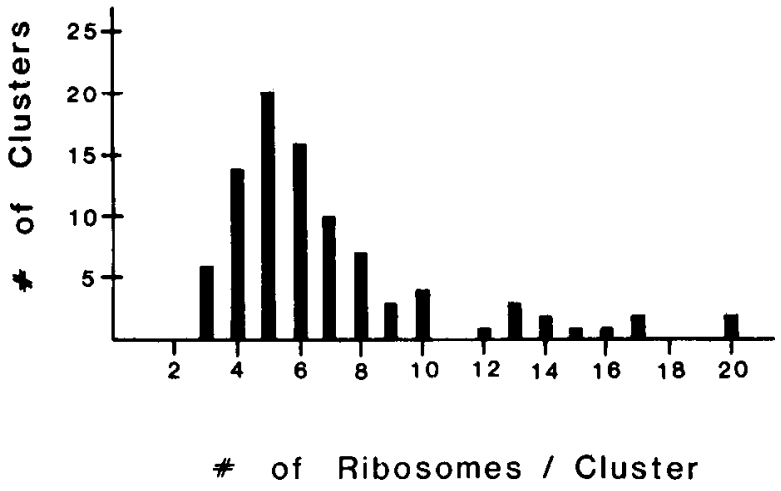

Figure 6. Frequency histograms of the number of ribosomes per cluster from the hippocampus proper. $A$, Polyribosomes beneath either identified or presumed spines. $B$, Polyribosomes within the dendrite proper that are not obviously associated with spines.

\section{Discussion}

The quantitative analyses of polyribosomes beneath and within spines suggest several generalizations. First, it is clear that many of the polyribosomes at the base of spines are associated with cisterns, whereas polyribosomes in spine heads are almost always free. Dendritic polyribosomes that are not apparently associated with spines are similar in most respects to the polyribosomes at the spine bases. Second, there appears to be some association between spine apparatuses and polyribosomes on cisterns. Spines with a spine apparatus are more likely to have underlying polyribosomes on cisterns than are spines without apparatuses. From another perspective, polyribosomes that are present at the base of spines with spine apparatuses are much more likely to be associated with a cistern than are polyribosomes beneath spines without a spine apparatus. Finally, there are usually fewer ribosomes per polyribosome in spine heads. Before discussing the implications of these observations, it is important to consider the quantitative methods with which the data were derived.

\section{Methods of analysis}

As noted above, the viewing of thick sections in stereo is a useful approach for providing information on 3-dimensional relationships between organelles that are close together (polyribosomes and cisterns, for example). One must be concerned, however, that some of the cisterns associated with particular clusters might lie out of the plane of the section containing the polyribosomes. In addition, counts of the number of ribosomes per cluster are potentially compromised by the fact that the clusters are large with respect to section thickness, and some of
A.

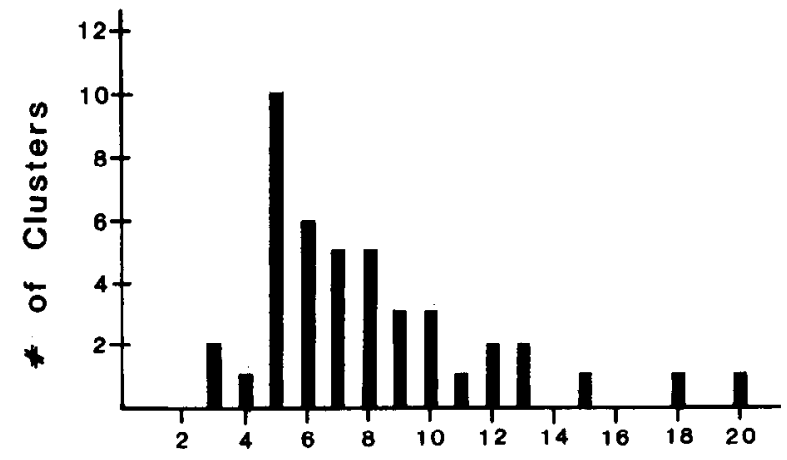

B.

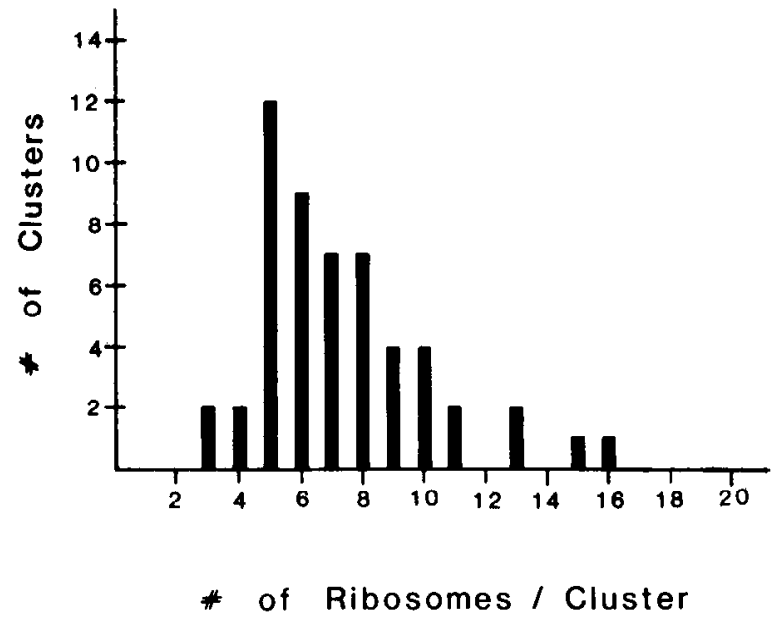

Figure 7. Frequency histograms of the number of ribosomes in cisternassociated and free polyribosomes (hippocampus). Only the polyribosomes beneath identified or presumed spines are evaluated in this graph. $A$, Polyribosomes on cisterns. $B$, Free polyribosomes.

the ribosomes of given clusters could lie in adjacent sections. With serial reconstruction, the analysis is not limited by section thickness, since the entire spine can be reconstructed. However, in counting the number of ribosomes per cluster using serial sections, one must be aware of the possible occurrence of double counts (one ribosome appearing in 2 sections).

Given the differences between the 2 methods of data analysis, the agreement between them is striking. Virtually the same proportion of polyribosomes were found to be associated with cisterns using both methods; furthermore, the counts of the number of ribosomes per cluster yielded almost identical data. The latter is particularly impressive, since one would expect the counts to be low in the case of the thick sections (because of loss of ribosomes in adjacent sections) and high in the case of serial reconstructions (because of possible double-counting).

\section{Comparison with previous studies}

There are few other studies with which the present study can be directly compared. Vaughn and Peters (1973) studied spines in layer 1 of the parietal cortex of the rat using serial reconstruction methods. They found that about $50 \%$ of the spines in the upper part of layer $1 \mathrm{had}$ a spine apparatus and that the proportion of spines with spine apparatuses increased with increasing depth in layer 1. Spacek (Spacek and Hartmann, 1983; Spacek, $1985 \mathrm{a}, \mathrm{b}$ ) reconstructed spines from pyramidal cells of the cerebral cortex and Purkinje cells of the cerebellum of young 
adult mice. In the cerebral cortex, $82 \%$ of all spines had ribosomes in the head (not necessarily polyribosomes as defined herein); $42 \%$ had ribosomes in the stalk, and $62 \%$ had ribosomes at the base (Spacek, 1985a). About $68 \%$ of the spines had a spine apparatus. In the cerebellum, 13\% of the spines had ribosomes in the head, and $22 \%$ had ribosomes at the base. Cerebellar Purkinje cells do not have well-devcloped spinc apparatuscs. The reasons for the differences in the incidence of polyribosomes and spine apparatuses are not clear, but may be due to the differences between cell types, to differences in the criteria used to identify polyribosomes and spine apparatuses, or to differences in the proximodistal location of the spines analyzed in each study.

\section{Significance of associations between polyribosomes, cisterns, and spine apparatuses}

The association between polyribosomes and cisterns is reminiscent of that between ribosomes and membranes of the rough endoplasmic reticulum. This invites the speculation that polyribosomes on cisterns synthesize the same type of proteins as do polyribosomes of the rough endoplasmic reticulum (i.e., membrane proteins or proteins for release). It is important to note, however, that it was relatively rare to find ribosomes that could definitely be said to be immediately apposed to a membrane; more often, the clusters appeared to lie adjacent to the cisternal membranes. Even so, the association suggests some functional relationship.

If the polyribosomes on cisterns do synthesize membrane proteins or proteins for release, the question arises as to whether these are glycoproteins that would require posttranslational processing. In this regard, it is of interest that polyribosomes are much more likely to be on cisterns when there is a spine apparatus present. From a purely morphological point of view, there is certainly some similarity between the spine apparatus and the Golgi apparatus of the cell body. Thus, an interesting possibility is that the spine apparatus might play a role in the posttranslational processing of proteins that were synthesized on the polyribosomes. This would also be consistent with previous speculation that the spine apparatus might play a role in synapse remodeling (Tarrant and Routtenberg, 1979; Dyson and Jones, 1984). Such a role would not exclude the possibility that the spine apparatus is also a calcium-sequestering organelle (Fifkova et al., 1983).

It is important to note that polyribosomes are most prevalent during times of synapse growth, whereas spine apparatuses are not common until the synapse is relatively mature (Adinolfi, 1972). If polyribosomes on cisterns, together with spine apparatuses, do represent a system for the synthesis and processing of proteins, these proteins may not be required until the synapse matures. Other classes of proteins (synthesized by polyribosomes that are not associated with spine apparatuses) may be important during periods of synapse growth. This provides evidence, albeit indirect, that different classes of proteins may be synthesized by the different types of polyribosomes beneath the synaptic site.

\section{References}

Adinolfi, A. M. (1972) The organization of paramembranous densities during postnatal maturation of synaptic junctions in the cerebral cortex. Exp. Neurol. 34: 383-393.

Bruning, J. L., and B. L. Kintz (1977) Computational Handbook of Statistics, Scott Foresman, Glenville, IL.
Dyson, S. E., and D. G. Jones (1984) Synaptic remodeling during development and maturation: Junction differentiation and splitting as a mechanism for modifying connectivity. Dev. Brain Res. 13: 125137.

Fifkova, E., J. A. Markham, and R. J. Delay (1983) Calcium in the spine apparatus of dendritic spines in the dentate molecular layer. Brain Res. 266: 163-168.

Fujiki, Y., R. A. Rachubinski, and P. B. Lazarow (1984) Synthesis of a major integral membrane polypeptide of rat liver peroxisomes on free polysomes. Proc. Natl. Acad. Sci. USA 81: 7127-7131.

Hall, C., L. C. Mahadevan, S. A. Whatley, T.-S. Ling, and L. Lim (1982) The polyadenylated RNA directing the synthesis of the rat myelin basic proteins is present in both free and membrane-bound forebrain polyribosomes. Biochem. J. 202: 407-417.

Manina, A. A. (1979) The synapses of the nervous system. Int. Rev. Cytol. 57: 345-383.

Palacios-Pru, E. L., L. Palacios, and R. V. Mendoza (1981) Synaptogenetic mechanisms during chick cerebellar cortex development. J. Submicrosc. Cytol. 13: 145-167.

Palade, $G$. (1965) Intracellular aspects of the process of protein synthesis. Science 189: 347-358.

Spacek, J. (1985a) Relationships between synaptic junctions puncta adhaerentia and the spine apparatus at neocortical axo-spinous synapses: A serial section study. Anat. Embryol. 173: 129-135.

Spacek, J. (1985b) Three-dimensional analysis of dendritic spines II. Spine apparatus and other cytoplasmic components. Anat. Embryol. 171: 235-243.

Spacek, J., and M. Hartmann (1983) Three-dimensional analysis of dendritic spines I. Quantitative observations related to dendritic and synaptic morphology in cerebral and cerebellar cortices. Anat. Embryol. 167: 289-310.

Steward, O. (1983a) Alterations in polyribosomes associated with dendritic spines during the reinnervation of the dentate gyrus of the adult rat. J. Neurosci. 3: 177-188.

Steward, O. (1983b) Polyribosomes at the base of dendritic spines of central nervous system neurons: Their possible role in synapse construction and modification. Cold Spring Harbor Symp. Quant. Biol. 48: 745-759.

Steward, O., and P. M. Falk (1985) Polyribosomes under developing spine synapses; growth specializations of dendrites at sites of synaptogenesis. J. Neurosci. Res. 13: 75-88.

Steward, O., and P. M. Falk (1986) Protein synthetic machinery at postsynaptic sites during synaptogenesis; a quantitative study of the association between polyribosomes and developing synapses. J. Neurosci. 6: $412-423$.

Steward, O., and W. B. Levy (1982) Preferential localization of polyribosomes under the base of dendritic spines in granule cells of the dentate gyrus. J. Neurosci. 2: 284-291.

Steward, O., and C. E. Ribak (1986) Polyribosomes associated with synaptic specializations on axon initial segments: Localization of protein-synthetic machinery at inhibitory synapses. J. Neurosci. 6: 3079 3085.

Steward, O., L. Davis, T. M. Reeves, and G. Banker (in press) Microcompartmentation of the protein synthesis machinery of neurons; polyribosome localization under postsynaptic sites. In Intrinsic Determinants of Neuronal Form, R. Lasek, ed., Allan R. Liss Inc., New York.

Tarrant, S. B., and A. Routtenberg (1977) The synaptic spinule in the dendritic spine: Electron microscopic study of the hippocampal dentate gyrus. Tissue and Cell 9: 461-473.

Tarrant, S. B., and A. Routtenberg (1979) Postsynaptic membrane and spine apparatus: Proximity in dendritic spines. Neurosci. Lett. 11: 289-294.

Vaughn, D. W., and A. Peters (1973) A three dimensional study of layer I of the rat parietal cortex. J. Comp. Neurol. 149: 355-370.

Warner, J. R., A. Rich, and C. E. Hall (1962) Electron microscopic studies of ribosomal clusters synthesizing hemoglobin. Science 138 . 1399-1403.

Westrum, L. E., D. H. Jones, E. G. Gray, and J. Barron (1980) Microtubules, dendritic spines, and the spine apparatus. Cell Tissue Res. 208: 171-181. 